\title{
Über die Entstehung von Dextrose aus Aminosãuren bei Phlorhizinglykosurie.
}

\author{
Von \\ A. J. Ringer und Graham Lusk. \\ (Aus dem physiol. Laboratorium der Cornell-University Medical College, New York.)
} (Der Redaktion zugegangen am 24. März 1910.)

$\left.\mathrm{Knopf}^{1}\right)$ gab einem Hund, der täglich 3 Injektionen von Phlorhizin erhalten hatte, $50 \mathrm{~g}$ Asparagin. Wenn man annimmt, daß das Asparagin vollständig resorbiert wurde, kann man berechnen, daß 20,55 g Dextrose daraus entstanden sei.

Stiles und $\mathrm{Lusk}^{2}$ ) gaben ein Verdauungsprodukt von Pankreas und Fleisch, das nur Aminosäuren enthielt, einem Hund, der Phlorhizin erhalten hatte, und fanden eine Ausscheidung von «Extra Dextrose», welche ungefähr $40 \%$ der eingegebenen Menge betrug. Diese Experimente stellten den synthetischen Ursprung des Zuckers aus Protein sicher fest.

$\mathrm{Halsey}^{3}$ ) fütterte Leucin an verschiedene Hunde, die Phlorhizin erhalten hatten, und kam zu dem Schluß, daß Leucin nicht in Dextrose verwandelt werden kann.

$\mathrm{Lusk}^{4}$ ) fand, daß, wenn $20 \mathrm{~g}$ Glutaminsäure eingegeben wurden, die Ausscheidung von Extra Dextrose im Harn 13,5 g betrug, und schloß, daß 3 Kohlenstoffatome der Glutaminsäure in Dextrose verwandelt wurden. Andere Experimente, die über Zufuhr von Aminosäuren bei Phlorhizinglykosurie ver-

1) Knopf, Archiv für experimentelle Pathol. und Pharmak., 1903, Bd. XLIV, S. 123.

2) Stiles u. Lusk, American Journ. of Physiol. 1903, Bd. IX, S. 380.

3) Halsey, American Jorn. of Physiol., 1904, Bd. X, S. 229.

4) Lusk, American Journ. of Physiol., 1908, Bd. XXII, S. 174. 
öffentlicht wurden, widersprechen sich in ihren Resultaten, ${ }^{1}$ ) und sind von geringem Wert wegen der ungeeigneten Methode, die Glykosurie zu erzeugen. Die richtige Methode ist die in einem einzigen Experiment von $\mathrm{Cremer}{ }^{2}$ ) angegebene, die in mehreren Arbeiten aus unserem Laboratorium ${ }^{3}$ ) weiter entwickelt wurde. Die jetzt befolgte Anordnung besteht darin, alle 8 Stunden $2 \mathrm{~g}$ Phlorhizin subcutan einzuspritzen, das in

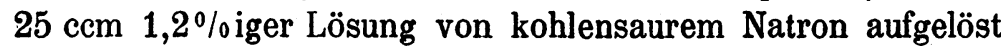
und auf $40^{\circ}$ erwärmt ist. Nachts kann die Zeit zwischen 2 Injektionen 10 Stunden dauern, aber dann sollte die 12 stündige Periode des Harnsammelns zwei Stunden nach der Morgeninjektion beendet sein. Dies sichert die Entfernung aller Dextrose, die während der langen Nachtzeit im Körper zurückgeblieben sein könnte. Folgende methodische Details müssen deshalb für die Sammlung des Urins in 12 stündigen Perioden beachtet werden:

$8 \mathrm{Uhr}$ vorm. $2 \mathrm{~g}$ Phlorhizin,

$10 \gg, \quad$ Katheter und Blase ausgespült. Die Periode beginnt.

3 Uhr nachm. 2 g Phlorhizin,

10 \ Katheter und Blase ausgespült. Die neue Periode beginnt, $2 \mathrm{~g}$ Phlorhizin.

$8 \mathrm{Uhr}$ vorm. $2 \mathrm{~g}$ Phlorhizin,

10 、 Katheter und Blase gespült. Ende der Periode, die neue Periode beginnt.

Der erste Tag der Behandlung mit Phlorhizin ist immer von einer Ausschwemmung von Zucker aus dem Körper begleitet. Am zweiten Tag erhält der Hund ein kaltes Bad und wird dann mehrere Stunden (6) in einem großen kalten Zimmer

1) $\mathrm{Zu}$ dieser Kategorie gehören die folgenden: Baer u. Blum, Hofmeisters Beiträge, 1907, Bd. X, S. 80; lbid., 1908, Bd. XI, S. 101; Glaesner u. Pick, Ibid., 1908, Bd. X, S. 473; Höckendorf, Biochem. Zeitschrift, 1909, Bd. XXIII, S. 281; Pflüger u. Junckersdorf, Pflügers Archiv, 1910, Bd. CXXXI, S. 201.

2) Cremer u. Ritter, Zeitschrift f. Biologie, 1892, Bd. XXIX, S. 256.

3) Reilly, Nolau u. Lusk, American Journ. of Physiol., 1898, Bd. I, S. 395; Stiles u. Lusk, Ibid., 1903, Bd. X, S. 67; Lusk, Zeitschrift f. Biologie, 1898, Bd. XXXVI, S. 82; Ibid., 1901, Bd. XLII, S. 31. 
bei $0^{\circ}$ gehalten, sodaß Kälteschauer eintreten und das Körperglykogen entfernt wird. ${ }^{1}$ ) Am folgenden Tag ist ein bestimmtes Verhältnis $\mathrm{D}: \mathrm{N}$ hergestellt, welches von Periode $\mathrm{zu}$ Periode während des Experiments fortdauert und die Basis für die Versuche bildet.

Viele Jahre lang ergab das Phlorhizin von Merck, 3mal täglich hungernden Hunden eingespritzt, am 3. Tag ein Ver-; hältnis $D: N$, welches beständig war und $3,65: 1$ betrug. Neuerdings hat das Phlorhizin dieser Firma dieses Verhältnis, nicht mehr gegeben. Glücklicherweise bleibt das Verhältnis bei dem neuen Präparat auch gleichmäßig, nur etwas niedriger, d. h. 3,2 bis $-2,7: 1$. Man kann noch mit dem Präparat arbeiten, obwohl die Unsicherheit in bezug auf dieses Verhältnis die Arbeit weniger befriedigend macht. Einige Phlorhizinpräparate von Merck erzeugen Tetanus und den Tod der Hunde. Solche Präparate muß man nicht verwenden, sondern sich neue verschaffen. Halsey ${ }^{2}$ ) machte schlimme Erfahrungen in dieser Hinsicht und die Schwierigkeit hat sich auch manchmal in unserer eigenen Arbeit gezeigt. $^{3}$ )

Es muß hinzugefügt werden, daß Embden und Salomon ${ }^{4}$ ) Glykokoll, Alanin und Asparagin Hunden ohne Pankreas gegeben haben und große Zunahme von Zucker im Harn beobachteten. Doch gaben sie keine Darlegung der genauen Menge Zucker, welche von jeder einzelnen Aminosäure erzeugt werden konnte. Nur bei einem ihrer Hunde wurde der Stickstoffgehalt im Harn bestimmt, und da bewies das Verhältnis $D: N$ eine unvollständige Entfernung des Pankreas.

\section{Versuche.}

In unseren Experimenten wurde Glykokoll, i-Alanin, Asparaginsäure, d-Glutaminsäure und Tyrosin Hunden gegeben,

1) Lusk, American Journ. of Physiol., 1908, Bd. XXII, S. 163.

2) Halsey, Sitzungsberichte der Ges. z. Beförderung der ges. Naturwissenschaften, Marburg, 1889, S. 102.

s) Lusk, Zeitschrift f. Biologie, 1901, Bd. XLII, S. 37.

4) Embden u. Salomon, Hofmeisters Beiträge, 1904, Bd. V, S. 507, und Bd. VI, S. 63. 
welchen Phlorhizin eingespritzt war, und die Menge des Zuckers, die von jedem erzeugt wurde, bestimmt. Gelegentlich sind auch Essigsäure, Glycerinsäure, Propylalkohol und eine Emulsion von Baumwollsamenöl gegeben worden, und ihr Einfluß auf die Ausscheidung von Zucker bestimmt worden.

In den eingegebenen Aminosäuren wurde eine Stickstoffbestimmung gemacht, um ihre Reinheit zu kontrollieren. Die Zahlen der Analyse der Harnbestandteile sind das Mittel aus Doppelanalysen. Stickstoff wurde nach Kjeldahl, Dextrose nach Allihn, Harnstoff nach Mörner-Sjöquist in der Modifikation von Spiro, ${ }^{1}$ ) Ammoniak nach Folin, Aceton allein nach Huppert-Messinger und zusammen mit $\beta$-Oxybuttersäure nach Shaffer ${ }^{2}$ ) bestimmt.

Die Aminosäuren wurden gewöhnlich in Wasser oder in Natriumbicarbonat gelöst und durch eine Schlundsonde eingegeben. Der Hund wurde dann in aufrechter Stellung gehalten, mit den Hinterfüßen auf dem Boden und sein Rücken an die Knie des Wärters gelehnt. Dies verhinderte Erbrechen, besonders nach Glykokoll.

Im allgemeinen vermehrte die Einführung von Aminosäuren bei Phlorhizinglykosurie die Stickstoffausscheidung im Urin, ersparte aber auch etwas Körpereiweiß. Dies widerspricht dem Verhalten einzelner Aminosäuren, wenn sie einem hungernden Hunde eingegeben werden. Sogar Glykokoll und Alanin, welche ganz in Dextrose übergeführt werden können, zeigten diese Ersparung. Daß die Aminosäuren nicht als solche im Urin erschienen, wird bewiesen dadurch, daß keine Zunahme von Reststickstoff auf die Einführung folgte. Als Reststickstoff wird bezeichnet die Differenz zwischen dem Gesamt- $\mathrm{N}$ und der Summe von Harnstoff- und Ammoniak-N. Die Menge der Dextrose, welche aus den verschiedenen Aminosäuren entsteht, kann nur berechnet werden, wenn man annimmt, daß die Aminosäure ganz absorbiert wird und daß Stickstoff daraus in dem Urin der 12 stündigen Periode nach der Eingabe ausgeschieden wird. Dies ist der Fall bei normalen Hunden.

1) Spiro, Hofmeisters Beiträge, 1907, Bd. IX, S. 481.

?) Shaffer; Journal of Biological Chemistry, 1908, Bd. V, S. 211. 
Wenn nun der Stickstoff der eingegebenen Aminosäure von dem Gesamtstickstoff der 12-Stundenperiode abgezogen wird, so erhält man die Menge Stickstoff, die der Körper verloren hat. Das Körper-N, vervielfacht mit dem Dextrosewert des Verhältnisses D : N, ergibt den Zucker, der sich aus der Zersetzung des Körpereiweißes dieser Zeit gebildet haben könnte. Wenn diese aus Körpereiweiß gebildete Dextrose abgezogen wird von der Menge der wirklich in dem Urin der Periode gefundenen Dextrose, so wird der Rest die Dextrose sein, die sich aus der eingegebenen Aminosäure gebildet hat. So wurden bei Hund II, Periode II, $20 \mathrm{~g}$ Glykokoll $(3,77 \mathrm{~g} \mathrm{~N})$ eingegeben. Gesamt-N im Urin war $11,1 \mathrm{~g}$, Körper-N war $(11,1-3,77 \mathrm{~g})$ $7,33 \mathrm{~g}, 7,33 \times 2,8=20,50 \mathrm{~g}$ Dextrose aus dem Körpereiweiß. $32,71 \mathrm{~g}$ Gesamt-Dextrose $-20,50 \mathrm{~g}=12,21 \mathrm{~g}$ Extra-Dextrose erhalten aus $20 \mathrm{~g}$ Glykokoll.

Bei diesen Berechnungen haben wir dasjenige Verhältnis $D: N$ angenommen, welches in den Hungerperioden als das vorherrschende festgestellt war. Wenn während einer auf die Einführung einer Substanz folgenden Periode das Verhältnis besonders hoch blieb, so wurde angenommen, daß die hier ausgeschiedene Dextrose aus der Substanz stamme, die in der früheren Periode eingegeben war. Bei Anwendung dieser Methoden haben wir folgende Resultate erhalten:

Nach Eingabe von $20 \mathrm{~g}$ Glykokoll

\begin{tabular}{|c|c|c|c|}
\hline \multicolumn{4}{|c|}{ Extra-Dextrose } \\
\hline Hund II & Periode & e II & 12,21 \\
\hline , III & , & IV & 14,77 \\
\hline III & , & $\mathrm{VI}+\mathrm{VIII}$ & 13,29 \\
\hline IV & , & IV & 11,12 \\
\hline V & , & II & 15,77 \\
\hline
\end{tabular}

Da $20 \mathrm{~g}$ Glykokoll ein Maximum von $16 \mathrm{~g}$ Dextrose geben können, ist es klar, daß Glykokoll ganz in Dextrose übergeführt werden kann.

Nach Eingabe von $20 \mathrm{~g}$ i-Alanin war die Extrazuckerausscheidung die folgende:

\begin{tabular}{|c|c|c|}
\hline \multirow[b]{2}{*}{ Hund I } & \multicolumn{2}{|c|}{ Extra Dextrose } \\
\hline & Periode II + III & 18,76 \\
\hline & IV & 18,78 . \\
\hline
\end{tabular}


Da angenommen werden kann, daß $20 \mathrm{~g}$ Alanin 20,22 g Dextrose ergeben, und da 92\% dieser Menge als Extrazucker im Urin erhalten wurde, so ist es klar, daß Alanin vollständig in Dextrose verwandelt werden kann. Diese Reaktion wird wahrscheinlich stattfinden, indem das Alanin unter gleichzeitiger Überführung in Milchsäure desamidiert wird, denn Mandel und Lusk ${ }^{1}$ ) haben gezeigt, daß Milchsäure vollständig in Dextrose übergeführt werden kann.

Die Ausscheidung von Extra-Dextrose nach Eingabe von $20 \mathrm{~g}$ Asparaginsäure war folgende:

$\begin{array}{ccc} & & \text { Extra-Dextrose } \\ \text { Hund II } & \text { Periode V } & 11,26 \\ \text { > III } & \text { II + III } & 13,43 \\ \text { > IV } & \text { VI } & 10,08 \\ \text { > V } & >\text { IV } & 14,92\end{array}$

Eine Berechnung zeigt, daß die folgende Menge Dextrose entstehen kann aus $20 \mathrm{~g}$ Asparaginsäure: aus 2 Atomen der 4 in seinem Molekül enthalten $\mathrm{C} 9,02 \mathrm{~g}$, von $3 \mathrm{C}$-Atomen $13,52 \mathrm{~g}$, aus $4 \mathrm{C}$-Atomen 18,04 g. Der Durchschnitt der erhaltenen Resultate zeigt, daß 3 von den 4 in Asparaginsäure enthaltenen Kohlenstoffatomen im Organismus in Dextrose verwandelt werden. Neben dem hier in Frage kommenden intermediären Prozeß kann man nur Vermutungen haben. Es könnte sein, daß die Asparaginsäure nach der Desamidierung in $\beta$ Milchsäure verwandelt wird, die in Dextrose übergeführt werden könnte.

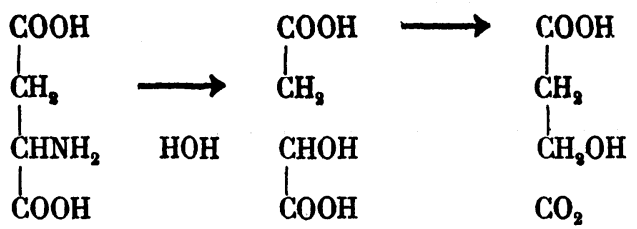

Die Angabe von Höckendorf, 2) daß Propylalkohol bei Phlorhizin-Glykosurie in Dextrose umgewandelt wird, aber Äthyl- und Butylalkohol nicht, schien von besonderem Interesse. Deshalb wurde Propylalkohol mit Äthylalkohol gemischt und in 2 Fällen eingegeben. In beiden war eine vermehrte Zuckerausscheidung. Die Resultate waren folgende:

1) Mandel u. Lusk, American Journ. of Physiol., 1906, Bd. XVI, S. 129.

2) Höckendorf, loc. cit. 
A. J. Ringer und Graham Lusk,

$\begin{array}{cccc} & & \text { Propylalkohol } & \text { Extra-Dextrose } \\ \text { Hund VIII Periode II } & 10 \mathrm{~g} & 5,24 \mathrm{~g} \\ \text {, IX } & \quad \text { VI } & \mathbf{5} & \mathbf{3}, 25\end{array}$

Hund VIII zeigte starke Vergiftungserscheinungen, Hund IX jedoch nicht. Die Verwandlung von Propylalkohol in Dextrose ist sehr wahrscheinlich nach diesen Experimenten, wenn man sie in bezug auf die von Höckendorf betrachtet. Dies zeigt wieder deutlich, mit welcher Leichtigkeit eine Kette von 3 Kohlenstoffatomen in Dextrose verwandelt werden kann.

Bei Eingabe von $20 \mathrm{~g}$ Glutaminsäure wurden folgende Resultate erhalten.

$\begin{array}{lcc} & \text { Extra-Dextrose } \\ \text { Hund } V & \text { Periode VI } & 13,16 \\ \text { Lusks } & \text { ) Hund I } & 13,46\end{array}$

Die Erklärung dieser Versuche wird durch die Tatsache ermöglicht, daß, wenn 3 C-Atome der Glutaminsäure Dextrose bilden, 12,24 g Extra-Dextrose im Harn erscheinen müßten, während, wenn $4 \mathrm{C}$-Atome Dextrose bilden, 16,32 g erlangt werden müßten. Die Resultate zeigen, daß 3 Kohlenstoffatome von den 5 in Glutaminsäure enthaltenen in Dextrose übergeführt werden können.

Die zwei oben genannten Resultate wurden erhalten, als das Verhältnis $D: N$ im Hungerzustand von Hund $V$ 2,6:1 war, während bei Lusks Hund I das Verhältnis 3,65:1 betrug. In bezug auf die Menge des Zuckers und Glutaminsäure bei Diabetes sind hiernach die Resultate unabhängig davon, ob das Verhältnis 3,65 oder 2,8:1 beträgt, wenigstens was die Glutaminsäure betrifft. Die biologische Theorie würde die Entfernung des Aminoradikals durch Hydrolyse fordern, und vielleicht würde eine hydrolytische Spaltung bei dem $\beta$-Kohlenstoffatom stattfinden, wie folgt:

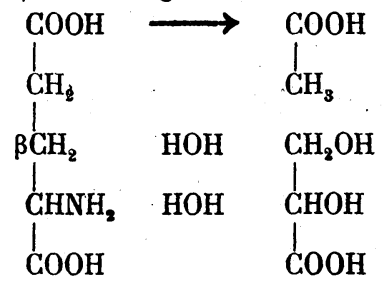

1) Lusk, American Journ. of Physiol., 1908; Bd. XXII, S. 176. 
Um die Möglichkeit dieser Theorie zu untersuchen, haben wir $10 \mathrm{~g}$ Natriumacetat dem Hund VI, Periode II, eingegeben und fanden, daß es ohne Einfluß auf die Zuckerausscheidung war, während Glycerinsäure, dem Hund II, Periode VII, und dem Hund IV, Periode VIII, beigebracht, eine beträchtliche Zunahme in der Zuckerausscheidung hervorbrachte. Erbrechen und Diarrhöe verhinderten quantitative Bestimmungen bei diesen letzterwähnten beiden Hunden.

Infolge dieser Resultate kann man auch schließen, daß Serin, welches durch Desamidierung in Glycerinsäure übergeht, in Dextrose umwandelbar ist.

In einem Fall wurden $10 \mathrm{~g}$ und in einem andern $7,5 \mathrm{~g}$ Tyrosin eingegeben, dem'Hund VII, Periode II, und demHund VIII, Periode IV. In keinem Fall konnte eine Entstehung von Zucker aus Tyrosin mit Sicherheit nachgewiesen werden, und im letzteren Fall bestätigte die starke Ausscheidung von $2,1 \mathrm{~g} \beta-0 x y b u t t e r-$ säure im Urin die Entdeckung von Baer und Blum, 1) daß dies ein normales Produkt der Tyrosinzersetzung ist.

Dreimal wurden etwa $10 \mathrm{~g}$ eines Esters von Glukosamin eingegeben, $\mathrm{C}_{6} \mathrm{H}_{11} \mathrm{O}_{5} \mathrm{NH} \cdot \mathrm{CO} \cdot \mathrm{C}_{2} \mathrm{H}_{5}$.

Diese Substanz wurde einem von uns (L.), von Professor Karl Spiro von Straßburg gegeben, und seiner Freundlichkeit verdanken wir die Gelegenheit, die Wirkung von Glukosamin bei Phlorhizin-Glykosurie zu prüfen. Das Material war dasselbe, das Forschbach ${ }^{2}$ ) Hunden ohne Pankreas und Meyer ${ }^{8}$ ) mit Phlorhizin gefütterten Kaninchen gegeben hatten. Forschbach wies darauf hin, daß, obwohl eine positive Zunahme der reduzierenden Kraft des Urins keinen Umsatz von Glukosamin in Dextrose im Organismus bedeute, doch ein negatives Resultat sicher bewiese, daß Glukosamin bei seiner Zersetzung nicht durch das Stadium einer Glukose hindurchgeht. Ein klares negatives Resultat zeigte der Hund VI, Periode V, während bei Hund VII, Periode IV, und bei Hund IX, Periode IV, anscheinend

1) Baer und Blum, Archiv f. exp. Pathologie und Pharmakologie, 1907, Bd. LVI, S. 96.

2) Forschbach, Hofmeisters Beiträge, 1906, Bd. VIII, S. 313.

s) Meyer, Hofmeisters Beiträge, 1907, Bd. IX, S. 134.

Hoppe-Seyler's Zeitschrift f. physiol. Chemie. LXVI. 
Zunahme der Dextrose im Harn stattfindet, welche aber von der Gegenwart des Glukosamins selber herrühren kann. Wir schließen deshalb mit Forschbach und Meyer, daß Glukosamin im Organismus nicht in Dextrose verwandelt wird. Schließlich gaben wir dem Hund IX, Periode VIII, $50 \mathrm{ccm}$ einer von Dr. L. H. Mills präparierten, feinen Fettemulsion, welche $66,4 \%$ Baumwollsamenöl enthielt. Diese war ohne Einfluß auf die Ausscheidung von Harnzucker, was durch das unveränderte D : N-Verhältnis bewiesen wird.

Im Verlauf der Arbeit wurden verschiedene Bestimmungen der Acetonkörper gemacht. Nur bei Tyrosin zeigte sich eine deutliche Zunahme dieser Körper. Einführung von Glykokoll, Asparagin- und Glutaminsäure verringerte gewöhnlich die Ausscheidung der Acetonkörper, sogar wenn Glykokoll fast vollständig in Traubenzucker verwandelt worden war. Vermehrte Diurese folgte immer auf die Einführung der Aminosäuren, und ob diese vermehrte Bewegung des Wassers durch den Körper die Verbrennung der Acetonkörper begünstigte oder nicht, ist eine offene Frage.

Was nun die Bildung von Zucker aus Fleisch anbetriff, so kann man seinen Ursprung jetzt annähernd bestimmen. Es ist festgestellt, daß bei einem mit Phlorhizin gefütterten Hund ${ }^{l}$ ) und bei einem diabetischen Menschen ${ }^{2}$ ) aus 100 Teilen zersetzten Eiweißes 58 Teile Dextrose hervorgehen können.

Osborne ${ }^{3}$ ) hat eine Analyse von Ochsenmuskel publiziert. In einem kürzlich in Boston gehaltenen Vortrag hat Osborne gezeigt, daß infolge der Ungenauigkeit der Fischerschen Estesmethode man ruhig die Zahlen für Alanin und Asparaginsäure verdoppeln kann. Die Arbeit von Parker und Lusk ${ }^{4}$ ) zeigte, daß $4 \%$ Glykokoll entstehen konnten durch die Zersetzung vøn

1) Reilly, Nolan u. Lusk, American Journ. of Physiol., 189, Bd. I, S. 395. Stiles u. Lusk, Ibid., 1903, Bd. X, S. 67.

2) Mandel u. Lusk, Deutsches Archiv für klinische Medizin, 19C', Bd. LXXXI, S. 472. S. 437.

3) Osborne u. Jones, American Journ. of Physiol., 1909, Bd. XXI/,

4) Parker u. Luusk, American. Journ. of Physiol., 1900, Bd.III, S. 47. 
Protein in einem hungernden Kaninchen. Einer der Verfasser (Ringer) ${ }^{1}$ ) nahm an einem Tag in seiner Nahrung $3 \mathrm{~g}$ benzoesaures Natron zu sich und dann 3 Tage lang $6 \mathrm{~g}$ täglich. Die Glykokollausscheidung stieg auf 3,$41 ; 3,55 ; 3,25$ und $3,48 \%$ des an diesen vier aufeinanderfolgenden Tagen zersetzten Eiweißes. Man kann ruhig berechnen, daß ungefähr $4 \%$ Glykokoll aus dem gewöhnlichen Fleischeiweiß entstehen.

Wenn man diese Betrachtungen anwendet auf die Entstehung des Zuckers aus Aminosäuren, so kann man folgende Tabelle aufstellen:

\begin{tabular}{|c|c|c|c|c|}
\hline & & $\begin{array}{c}\text { Osbornes } \\
\text { Analyse }\end{array}$ & $\begin{array}{c}\text { Wieder } \\
\text { berechnet }\end{array}$ & $\begin{array}{l}\text { Dextrose- } \\
\text { entstehung }\end{array}$ \\
\hline Glykokoll . . & . . . . . & 2,06 & 4,0 & 3,2 \\
\hline Alanin . . . & ..... & 3,72 & 7,5 & 7,5 \\
\hline Asparaginsäure & ...... & 4,51 & 9,0 & 6,1 \\
\hline \multirow[t]{2}{*}{ Glutaminsäure } & \multirow[t]{2}{*}{$\cdot \cdot \cdot \cdot$} & 15,49 & 15,5 & 9,5 \\
\hline & & 25,78 & 36,0 & 26,3 \\
\hline
\end{tabular}

Es ist klar, daß aus diesen vier Säuren 26 oder beinahe die Hälfte der 58 Teile der Dextrose, die von Eiweiß herstammt, entstehen können. In andern Worten: 45\% der gesamten Zuckerausscheidung bei Diabetes kann aus den 4 genannten Säuren entstehen.

\section{Zusammenfassung.}

Glykokoll und Alanin können ganz in Dextrose verwandelt werden. Drei Kohlenstoffatome der Asparaginsäure und der Glutaminsäure können in Dextrose verwandelt werden. Tyrosin gibt keine Dextrose, vermehrt aber die $\beta$-Oxybuttersäure im Harn. Glukosamin gibt keine Dextrose. Glycerinsäure und Propylalkohol geben Dextrose, aber Essigsäure gibt keine.

1) Chittenden, Long u. Herter, The influence of sodium benzoate. U. S. Dept. of Agriculture, 1909, Report 88, S. 676. 
A. J. Ringer und Graham Lusk,

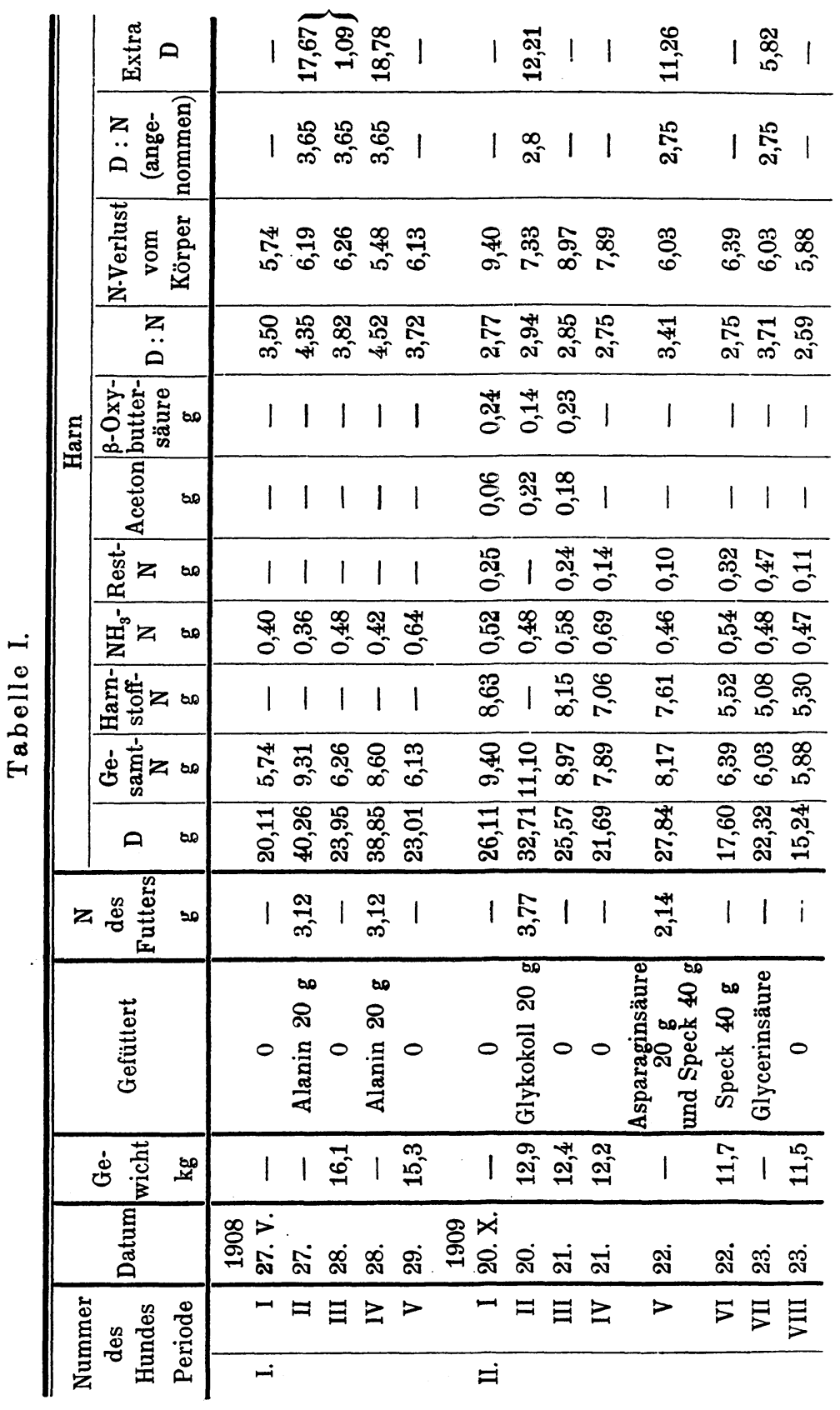


Über die Entstelhung von Dextrose aus Aminosäuren.

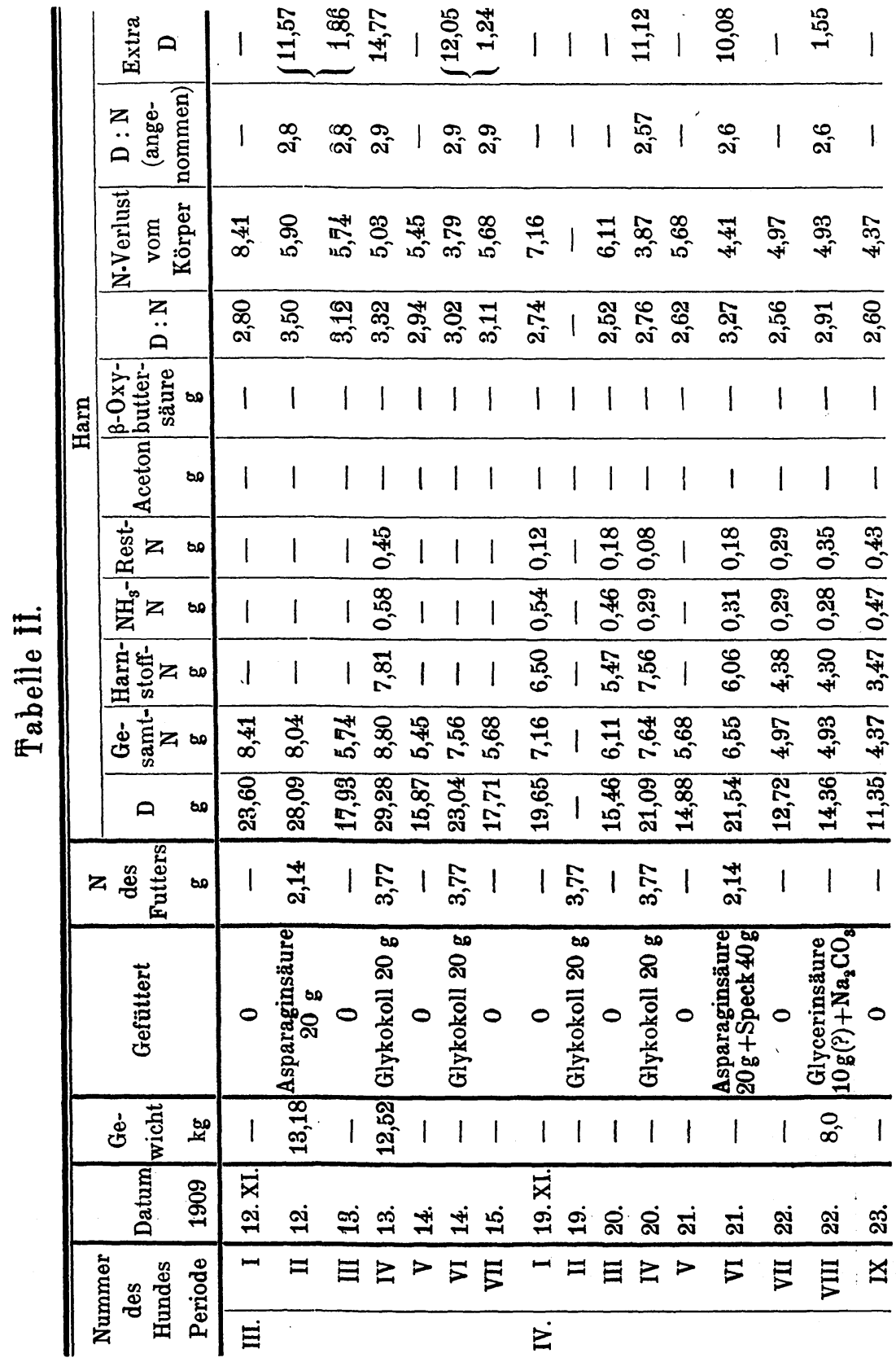




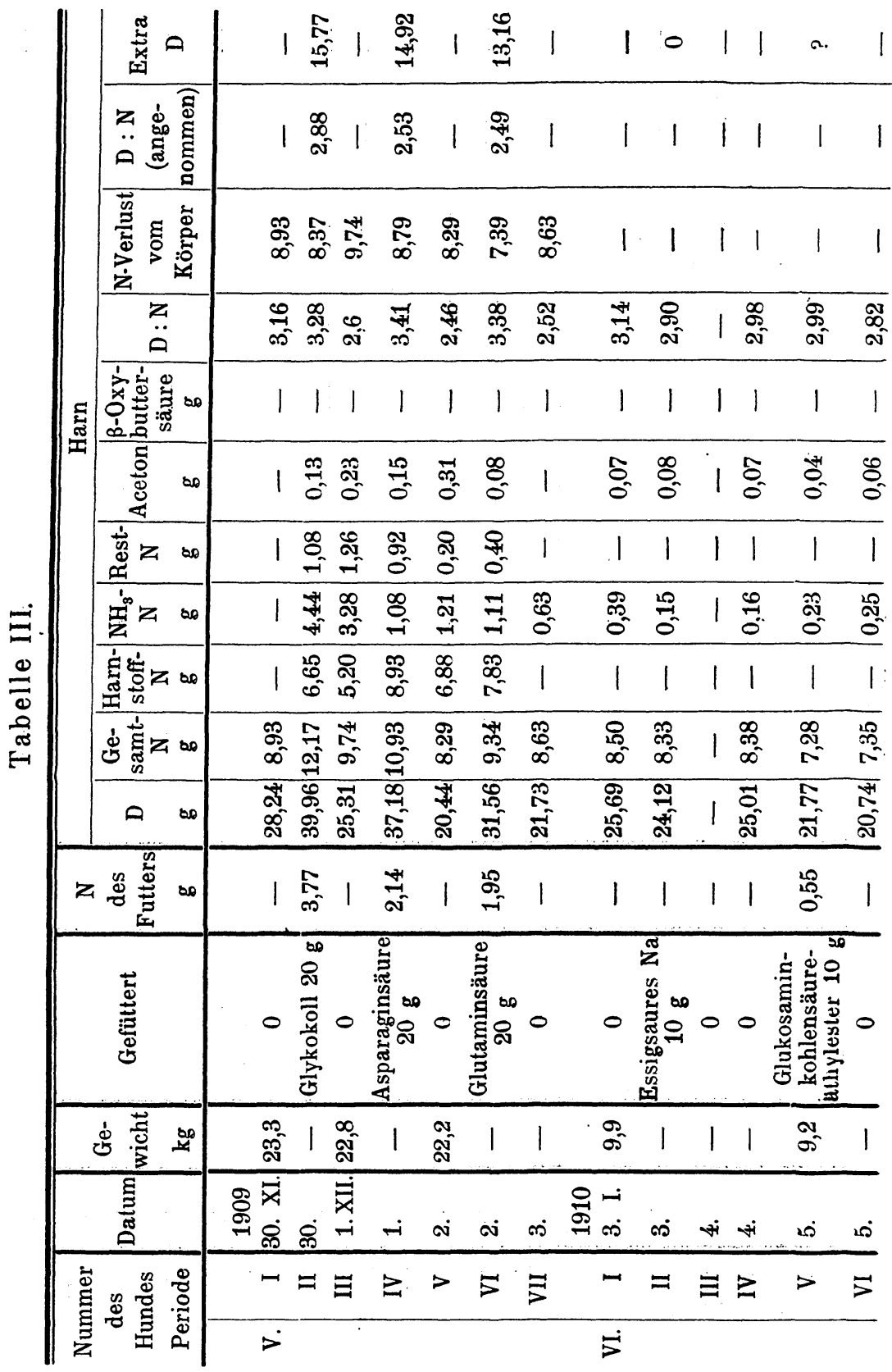


Ửber die Entstehung von Dextrose aus Aminosăuren.

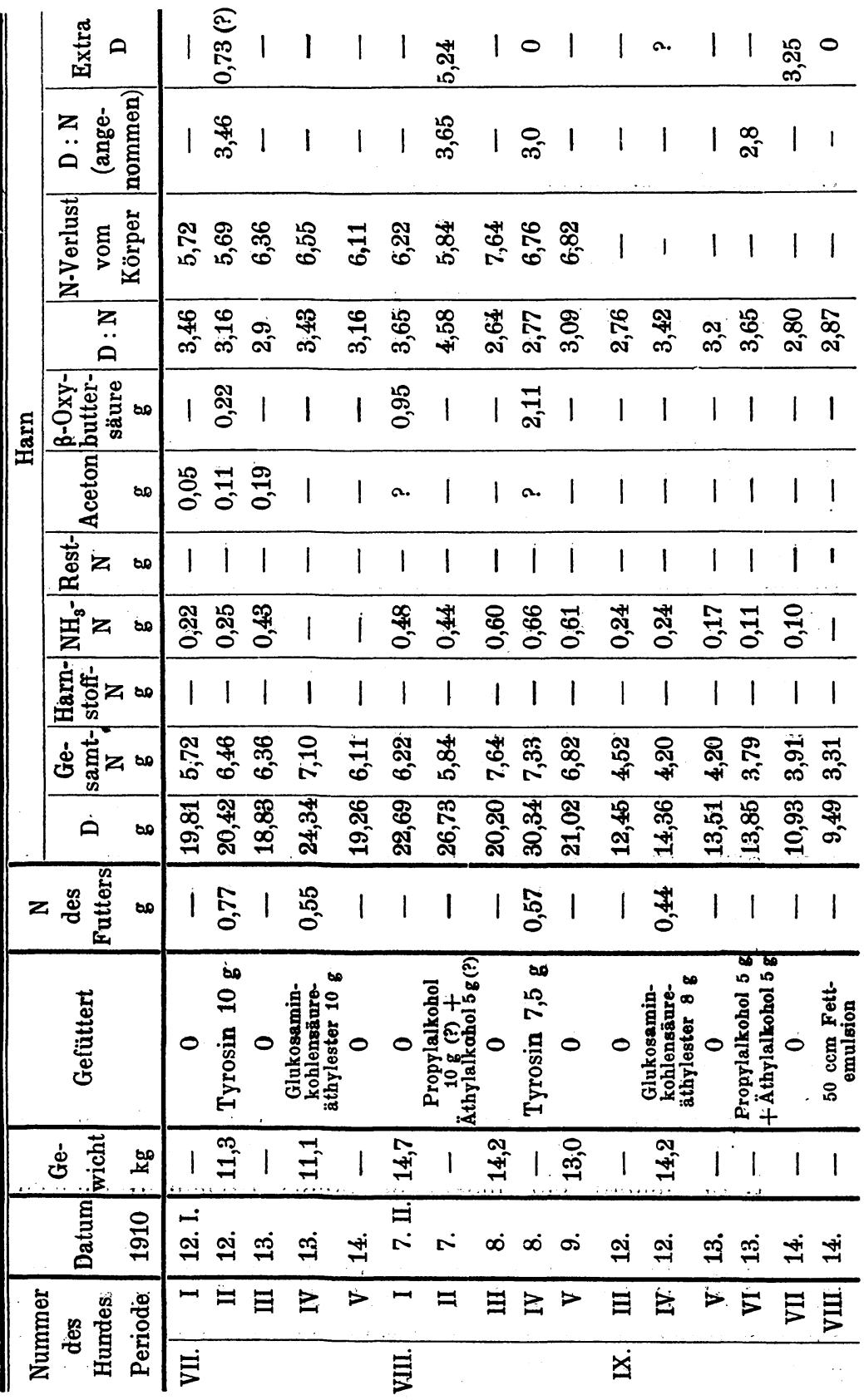

